\title{
CONVERGENCIA DiNÁMICA DE LOS INTERCAMBios COMERCIALES ENTRE CHINA y MÉXico, I 993-20 I 9
}

\author{
Xuedong Liu y Gerardo Covarrubias ${ }^{\mathrm{a}}$
}

Fecha de recepción: 20 de agosto de 2020. Fecha de aceptación: 3 de marzo de 2021.

$$
\text { https://doi.org/10.22201/iiec.20078951e.2021.206.69779 }
$$

Resumen. El objetivo de este trabajo es examinar la estabilidad dinámica del proceso estocástico dado por los intercambios comerciales entre México y China, en el periodo 1993-2019, a través de la aplicación de pruebas de estacionariedad y de la estimación de un modelo autorregresivo tanto en cifras anuales como mensuales. A pesar de las fluctuaciones persistentemente registradas en la serie del análisis, se observa que la varianza presenta una reducción significativa en todo el periodo, en tanto que la solución de las ecuaciones resultantes confirma que los flujos comerciales efectuados entre los dos países convergen en un nivel de estabilidad de 17.5, medidos por la proporción entre importaciones y exportaciones realizadas por México con el país asiático.

Palabras clave: intercambios comerciales; varianza; raíz unitaria; convergencia; estabilidad dinámica.

Clasificación JEL: F14; C01; C02; C13; C55; C62.

\section{DYNAMIC CONVERGENCE OF TRADE EXCHANGES Between China And MeXico, I993-20i9}

\begin{abstract}
This article examines the dynamic stability of the stochastic process, using the trade exchanges between Mexico and China during the period 1993-2019. The methodology consists of applying stationarity tests and estimating an autoregressive model in both annual and monthly figures. Despite persistent fluctuations registered in the series of the analysis, the variance presents a significant reduction across the study period, while the solutions to the resulting equations confirm that the trade flows between the two countries converge to a stability level of 17.5, measured using the ratio between Mexican imports and exports with China.
\end{abstract}

Key Words: trade flows; variance; unit root; convergence; dynamic stability.

\footnotetext{
${ }^{a}$ Universidad Nacional Autónoma de México (UNAm), Facultad de Estudios Superiores Aragón, México. Correos electrónicos: xdong@comunidad.unam.mx y gerardo_covarrubias_lopez@hotmail. com, respectivamente. Se agradece y se reconoce plenamente al Programa de Becas Posdoctorales de la UNAM en la elaboración de esta investigación. Este estudio forma parte del Proyecto PAPIIT IN311020, para el periodo de 2020-2022, financiado por la Dirección General de Asuntos para Personal Académico (DGAPA) de la UNAM.
} 


\section{INTRODUCCIÓN}

Desde hace más de 30 años, México ha sostenido saldos deficitarios en sus intercambios comerciales con China de manera constante y con una tendencia alcista, de acuerdo con cifras reveladas por la Secretaría de Economía (Secretaría de Economía [sE], 2020). Tan sólo en el periodo de 1993 a 2019, la tasa de crecimiento anual fue de $23.1 \%$ en promedio, al pasar de un monto de US\$341.7 a 75922.0 millones; es decir, el déficit registrado por México en la balanza comercial con el país asiático se multiplicó por 222 durante los últimos 26 años.

Todo indicaría que la cifra pudiera incrementarse aún más con el transcurso del tiempo en términos absolutos. Por esta razón, en este trabajo se tiene como objetivo analizar la estabilidad dinámica de este proceso estocástico, referente a términos relativos de los intercambios comerciales sino-mexicanos por medio de la convergencia dinámica, la cual se entiende por el hecho de que la trayectoria temporal del indicador en el análisis registraría una tendencia hacia un nivel particular, a pesar de que la definición formal se puede ver más adelante. Con los resultados obtenidos se espera describir la relación comercial entre ambas naciones en términos tanto cualitativos como cuantitativos, sobre todo, para contribuir a la formulación de políticas comerciales a futuro para México de manera inteligente y provechosa con su segundo socio comercial, una economía preponderante dentro del entorno global contemporáneo.

El análisis se realiza a partir de distinguir la información estadística relacionada con los intercambios comerciales entre las dos naciones, en términos tanto absolutos como relativos, en el periodo 1993-2019. Por un lado, el saldo deficitario en términos absolutos definitivamente registra una tendencia creciente para México en el transcurso del tiempo. Por otro lado, las mismas cifras, pero en términos relativos y expresadas por la proporción entre las importaciones $(\mathrm{M})$ y las exportaciones $(\mathrm{X})$ mexicanas efectuadas con su segundo socio comercial $(\mathrm{M} / \mathrm{X})$, se encuentran en una senda aparentemente decreciente $y$ con tendencia a un posible equilibrio.

Teóricamente los modelos de series temporales que se aplican en este estudio tienen la finalidad de realizar un pronóstico del patrón dinámico del comportamiento temporal o la extrapolación de un proceso estocástico (Harvey, 1993; Maddala, 2001; Guerrero, 2011; Enders, 2015), por ello, la trayectoria temporal de una variable parte de las características de sus componentes predictibles y de sus rezagos en el tiempo como núcleo del análisis dinámico. Para el caso de este estudio, la proporción M/X se representa por una ecuación en 
diferencias en función de sus propios rezagos, y los resultados obtenidos revelan un comportamiento de convergencia dinámica y con tendencia a la baja, además de que las fluctuaciones encontradas en esta variable presentan una tendencia persistentemente descendente.

De lo anterior, se puede afirmar que a pesar de que el comercio bilateral entre México y China se caracteriza por un desequilibrio, se acentúa cada vez más en favor del país asiático desde 1993 a la fecha. Asimismo, durante este periodo, también se han reportado altibajos frecuentes, ya que, en promedio, el valor total de las importaciones procedentes de China representa 13.5 veces el valor de las exportaciones recíprocas, ambas a cifras anuales. En casos extremos, se puede observar que en 1997 esta brecha se amplió hasta su punto máximo de 27.2, mientras que en 1993, la misma cifra fue de 8.6 como mínimo, con una varianza de 15.2. De acuerdo con las proyecciones derivadas del modelo propuesto, la variable tendería a converger hacia un nivel de 17.5 como el punto del equilibrio.

El presente estudio se compone por cinco secciones, incluida esta introducción. En la segunda, se ofrece una revisión breve sobre los intercambios comerciales registrados entre las dos naciones durante los últimos 26 años; en seguida se muestran las estimaciones de las varianzas correspondientes en sus distintas modalidades y en diferentes lapsos, mismos que van desde 4 hasta 10 años para constatar que las fluctuaciones de la variable han registrado una inminente tendencia a la baja, lo que indica un vínculo entre los hechos registrados y la metodología utilizada en este estudio en el sentido de que los flujos comerciales entre las dos economías habrían encaminado a una senda cada vez más estable. En la cuarta sección, se observa que a través de la aplicación de la prueba de raíz unitaria de Dickey-Fuller Aumentada (DFA) se obtuvo que la serie $\mathrm{M} / \mathrm{X}$ arroja una tendencia fluctuantemente decreciente sin estacionariedad debido a la existencia de una caminata aleatoria en las cifras anualizadas desde 1993 hasta 2019. En tanto que la misma serie, pero medida con base mensual, registró un comportamiento dinámico convergente, con la particularidad de que no presenta raíz unitaria y, por lo tanto, es estacionaria. Finalmente, con base en los resultados obtenidos en las pruebas de raíz unitaria y los cálculos de varianza en sus distintas modalidades, se realizaron los pronósticos pertinentes para proyectar el comportamiento futuro de los intercambios comerciales entre las dos economías. Asimismo, se presentan las conclusiones en la última sección. 


\section{DEBATES SOBRE LOS INTERCAMBIOS COMERCIALES ENTRE CHINA Y MÉXICO, 1993-2019}

El comercio bilateral sostenido entre las dos economías comenzó a llamar la atención, en ámbitos tanto gubernamentales como académicos en la última década del siglo pasado, cuando México impuso cuotas compensatorias contra una canasta masiva de productos importados desde China ante su presencia creciente en el mercado doméstico nacional. Desde entonces, las medidas instrumentadas por las autoridades mexicanas para tratar de reducir el volumen de llegada de esos productos no han cesado.

\section{Instrumentos nuevos para aliviar el desequilibrio de la balanza comercial}

Durante el periodo de estudio se agregaron ciertas políticas ante la "amenaza" de que la competencia de los bienes hechos por el país asiático se encuentra vigente no sólo en el mercado interno mexicano, sino también en el mercado externo, sobre todo con Estados Unidos, hacia donde se destina usualmente más del $80 \%$ de la exportación de productos mexicanos, ante el eminente ingreso de China a la Organización Mundial del Comercio (омC) a finales de 2001.

Entre estos nuevos instrumentos destaca el convenio firmado por los dos gobiernos que se reservó en el Protocolo de Adhesión de China a la omc en diciembre de 2001, en el cual México mantenía cuotas compensatorias sobre un total de 953 fracciones arancelarias por un lapso de seis años. De acuerdo con la Secretaría de Economía (Secretaría de Economía [SE], 2007), el convenio logrado entre China y México contempla que el país asiático está obligado a adoptar reglas internacionalmente acordadas en la oMc para regir sus prácticas comerciales en materia de importaciones y exportaciones. Como parte de las negociaciones, México tendría un periodo de transición de seis años para implementar un programa de ajuste y competitividad en la industria a través de la aplicación de cuotas compensatorias a los 21 sectores, que incluyen bicicletas, calzado y sus partes, candados de latón, coches para transportes de niños, cerraduras de pomo y perillas, conexiones de hierro maleable, textiles, juguetes, lápices, prendas de vestir, entre otros.

El acuerdo también contempla que las medidas antidumping que en su momento aplicaba México "a productos chinos durante los seis años siguientes a la adhesión de China, las medidas actuales de México no se someterán 
a las disposiciones del Acuerdo sobre la OMC ni a las disposiciones sobre medidas antidumping" (DOF, 2007, p. 11). Es decir, estas mismas medidas no podrán ser cuestionadas ante un panel de solución de controversias de la OMC por un periodo de seis años a partir del ingreso de China a esa organización, las cuales se aplican para más de 1300 productos chinos.

Al 1 o de junio de 2008, las dos autoridades acordaron de nuevo que se mantuviera vigente la aplicación de las cuotas compensatorias para un grupo de 204 de las 953 fracciones arancelarias que México tenía la obligación de eliminar hasta el 11 de diciembre de 2011, como medida de transición (sE, 2008).

A diferencia de varios países latinoamericanos, México hasta la fecha no solamente no ha reconocido a la economía china como una economía de mercado, sino que se ha negado a adoptar una postura de transición en la que, en lugar de utilizar los precios o los costos de productos similares en terceros países, utilice los más parecidos a los que se aplican en el interior de China, y así determinar si el país asiático está realizando las exportaciones con prácticas de dumping con sus productos. De manera similar, también serviría para definir que los exportadores chinos reciben subvenciones o subsidios oficiales. Con base en estas comparaciones, se determinan los precios de referencia de importación de productos procedentes de China.

\section{Evaluación del desequilibrio de la balanza comercial de México con China}

Entre los numerosos estudios dedicados al tema, desde finales del siglo pasado hasta la actualidad, existen tanto acuerdos como desacuerdos con respecto a las características registradas en los intercambios comerciales entre las dos economías. Algunos autores sostienen que, en el comercio de México con Estados Unidos y China, existe una débil integración intraindustrial con el país asiático y una mayor integración con Estados Unidos, aunque decreciente (Cárdenas y Dussel, 2011; López y Rodil, 2019); además de estimaciones de carácter empírico donde se concluye que las exportaciones de México hacia Estados Unidos decrecieron rápidamente como resultado de la competencia directa con China (Jenkins y Dussel, 2009; Gallagher y Porzecanski, 2010; Gallagher y Dussel, 2013).

En primer lugar, se reconoce ampliamente y de manera consensada que los productos fabricados en China cuentan con múltiples y eminentes ventajas competitivas que van desde insumos, bienes intermedios y bienes de capital 
en comparación con sus similares mexicanos, tanto en el mercado doméstico de México como en los de exportación. Al respecto, cifras de la SE indican que desde 1993 a la fecha los intercambios comerciales efectuados por las dos economías arrojan saldos deficitarios con tendencia creciente para México, al pasar de US\$341.7 millones en 1993 a US\$75 922.0 millones en 2019 (sE, 2020).

$\mathrm{Al}$ revisar los comportamientos registrados por productos procedentes de ambas naciones en el mercado de Estados Unidos se llega a la misma conclusión. Pues desde 1994, a pesar de contar con beneficios por ser parte del Tratado del Libre Comercio de América del Norte (TLCAN), los productos de México perdieron una parte importante de su participación en el mercado de Estados Unidos ante la creciente presencia de similares chinos. De acuerdo con las cifras registradas por United States Census Bureau (2015), en 1993 cuando el TLCAN todavía no entraba en vigencia, la participación de productos chinos en el mercado estadounidense era de $5.43 \%$ contra la de los productos mexicanos de $6.87 \%$; es decir, se tenía una diferencia de $1.44 \%$ en favor de la economía mexicana.

Es importante mencionar que esta ventaja creció hasta 2000 y alcanzó su nivel máximo de $2.95 \%$, y las participaciones fueron de 11.16 y $8.21 \%$, respectivos para México y China. No obstante, una vez que China logró su ingreso a la OMC, se registraron modificaciones considerables al revertir las ventajas competitivas entre productos del país asiático y similares de México. Ante el crecimiento casi estancado, e incluso negativo en algunos años, de las exportaciones mexicanas hacia el mercado estadounidense, China desplazó a México en la segunda posición como proveedor del país económicamente más grande del mundo. ${ }^{1}$

Respecto al periodo 1993-2019, la estructura de comercio bilateral entre los dos países fue modificada, en su mayoría pasó desde productos de consumo generalizado hacia bienes de consumo intermedio y de capital. En otras palabras, el abrumador déficit registrado por México en su balanza comercial con China se debe cada vez más a las importaciones crecientes de artículos requeridos para su procesamiento y su integración a las cadenas nacionales, y que posteriormente se destinan a satisfacer la demanda de los mercados tanto doméstico como externo. Derivado de la modificación en la estructura del

1 Al iniciar el nuevo milenio varios sucesos, además del ingreso de China a la omc, la firma de acuerdos de libre comercio por parte de Estados Unidos con otros países, particularmente con algunos de Centroamérica y del Caribe, también propiciaron la pérdida relativa de las ventajas competitivas para las exportaciones mexicanas hacia Estados Unidos (Espinosa y Serra, 2005). 
comercio bilateral entre las dos economías, surgieron opiniones diversas e incluso controvertidas respecto a los desequilibrios en la balanza comercial entre China y México a partir de los distintos ángulos de discusión. A continuación, se presenta una breve semblanza al respecto.

Desde el punto de vista teórico, el ajuste en la estructura de los bienes involucrados en el comercio bilateral entre China y México durante las últimas tres décadas implicaría dos consecuencias. Por un lado, se podría propiciar una sustitución de importaciones procedentes de Estados Unidos por las de China. Por otro lado, las empresas orientadas a las exportaciones que operan en México tendrían la oportunidad de incrementar su eficiencia y productividad considerando que las importaciones chinas son más baratas que sus equivalentes de otras fuentes básicamente de Estados Unidos. En la medida en que las exportaciones chinas a México representan insumos de bajo costo y de mejor calidad para las empresas mexicanas, estas últimas deberían poder exportar a un precio más competitivo y, en consecuencia, ganar mayor participación en el mercado norteamericano. Sin embargo, de acuerdo con el estudio realizado por Dussel y Gallagher (2014), en un total de 53 sectores de mercado, Estados Unidos está perdiendo participación en favor de China, sectores en los que, de manera análoga, México pierde participación en el mercado norteamericano y también en beneficio de la economía asiática; por ejemplo, en sectores como calzado, juguetes, productos textiles, artículos de confección y material eléctrico y electrónico (Oropeza, 2006). En promedio, México había conquistado $14 \%$ del mercado de importación estadounidense en esos sectores en 2000, pero su participación se redujo al $9 \%$ en 2009.

En el mismo sentido, otros estudiosos argumentan que los cálculos realizados sobre el modelo de exportación mexicano, que requiere cada vez mayor participación de bienes chinos, indican que "ello va en detrimento de la competitividad, productividad y el mercado interno nacional" (De la Cruz y Veintimilla, 2014, p. 498). Además, el no incrementar la competitividad ha provocado una caída en el salario y un aumento en el déficit que México tiene con China. Así, mantener la política de fincar al comercio exterior como el motor de crecimiento de la economía mexicana es insuficiente, y en consecuencia, es prioritario desarrollar los sectores y regiones estratégicas de la economía mexicana (De la Cruz y Veintimilla, 2014).

Para otros, además de considerar la negatividad del creciente desequilibrio registrado en la balanza comercial entre la nación azteca y la asiática, todavía la catalogan como insostenible en el largo plazo. En este sentido, se tendrían que explorar y encontrar alternativas con el propósito de evaluar en tres dimensiones: como vehículo para elevar la competitividad regional, como estrategia de 
integración regional y como estrategia para impulsar el crecimiento (Villareal, 2014); aumentar las exportaciones mexicanas (a través de políticas de incentivos) e intentar atraer inversiones en la planta productiva nacional. "Por lo tanto, es importante un replanteamiento integral de las relaciones económicas sino-mexicanas" (Ley, 2012, p. 61). Asimismo, se requiere comenzar a convertir en realidad las declaraciones oficiales sino-mexicanas de que las relaciones entre ambos países son de carácter estratégico, a lo que se agregó en 2013 el adjetivo de "integral"; es decir, que son relaciones que por definición discursiva se trata de una asociación estratégica integral (Anguiano, 2016).

Sin embargo, contrario a los puntos de vista anteriores, la Secretaría de Relaciones Exteriores de México a través de su Embajada en China ha externado opiniones favorables a los intercambios comerciales realizados entre ambas economías. De acuerdo con los pronunciamientos, "una creciente complementariedad que nos ayuda a ser más competitivos en el mercado internacional, China es hoy nuestro segundo socio comercial, con intercambios cercanos a los US\$75 mmd en 2016; la segunda fuente de nuestras importaciones; y el tercer destino de nuestras exportaciones" (Embajada de México en China, 2015). Por su parte, México se mantiene como el primer socio comercial de China en América Latina.

En este contexto, Limas (2019) consideró que la mayor parte de los bienes importados de China por México contribuyen a la estructura productiva y exportadora de este último, principalmente la vinculada con Estados Unidos. Sin las importaciones precedentes de China muchas industrias mexicanas simplemente no podrían funcionar o serían mucho menos competitivas.

Además, se comenta que a pesar de que el déficit comercial con China creció prácticamente todos los años desde 1993, el comercio exterior de México se ha mantenido muy cerca del equilibrio desde la crisis de 1995. El déficit que se registró en la balanza comercial con China se ha visto compensado con creces por el superávit que se tiene con Estados Unidos (Ruíz, 2004; Zhang, 2013; Ortiz, 2011; Zottele y Santiago, 2015). De hecho, en la mayoría de las ocasiones, las importaciones en México son realizadas por transnacionales grandes cuya finalidad es regresar a mercados internacionales, lo que todavía podría aliviar posibles efectos negativos causados por ajustes cambiarios (Liu, 2012). Lo más importante de todo esto es que el déficit con China nunca ha sido un factor de desequilibro macroeconómico en México hasta la fecha, aunque en la balanza comercial se requiere un estudio más completo y profundo todavía (Limas, 2019). 


\title{
3. CARACTERÍSTICAS DE LOS INTERCAMBIOS COMERCIALES ENTRE CHINA Y MÉXICO, 1993-2019
}

\begin{abstract}
$\mathrm{Al}$ revisar el comportamiento registrado en los intercambios comerciales sostenidos entre los dos países de 1993 a 2019, principalmente ante la fuerte participación de China en las importaciones de productos pertenecientes a los capítulos 27, 84, 85 y 87 del Sistema Armonizado de Designación y Codificación de Mercancías, ${ }^{2}$ destacan dos particularidades interesantes. En primer lugar, el saldo deficitario creciente para México, aunque el ritmo de crecimiento se frenó a partir de 2008, debido a las causas ya conocidas de la crisis económica mundial; y en segundo lugar, la estabilización de la brecha entre importaciones y exportaciones a pesar de las fluctuaciones persistentes.
\end{abstract}

\section{Saldo deficitario creciente de México en su balanza comercial con China}

En la figura 1 se presenta un panorama general sobre los intercambios comerciales sostenidos por China y México durante el periodo 1993-2019. Al visualizar los comportamientos de importaciones, exportaciones y saldos deficitarios registrados por México con su segundo socio comercial, sobresale que una tasa del crecimiento acelerado que cubre prácticamente la mayor parte del lapso de análisis al menos hasta 2010, y posteriormente el mismo indicador se ha frenado dentro de un dígito hasta la fecha.

Como se puede observar, desde 1993 hasta 2010, la tasa anual de crecimiento de los saldos deficitarios registrados por México en su balanza comercial con China fue de $32.6 \%$ en promedio; mientras que para el periodo 2010-2019, la cifra respectiva fue de 6.7\%. Esta particularidad también se puede constatar a través de comparar el número de años requeridos para duplicar el volumen de los distintos indicadores relacionados con los intercambios comerciales entre los dos países. Concretamente, antes de 2008, tanto el volumen total como importaciones, exportaciones y saldo deficitario arrojados por México en su balanza comercial con China, se duplicaba en un

2 De acuerdo con este sistema, el capítulo 84 comprende: reactores nucleares, calderas, máquinas, aparatos y artefactos mecánicos; partes de estas máquinas o aparatos; el capítulo 85: máquinas, aparatos y material eléctrico, y sus partes; aparatos de grabación o reproducción de sonido, aparatos de grabación o reproducción de imagen y sonido en televisión, y las partes y los accesorios de estos aparatos; y el capítulo 87: vehículos automóviles, tractores, velocípedos y demás vehículos terrestres; sus partes y accesorios. 
Figura 1. Comercio bilateral entre China y México, 1993-2019 en millones de dólares

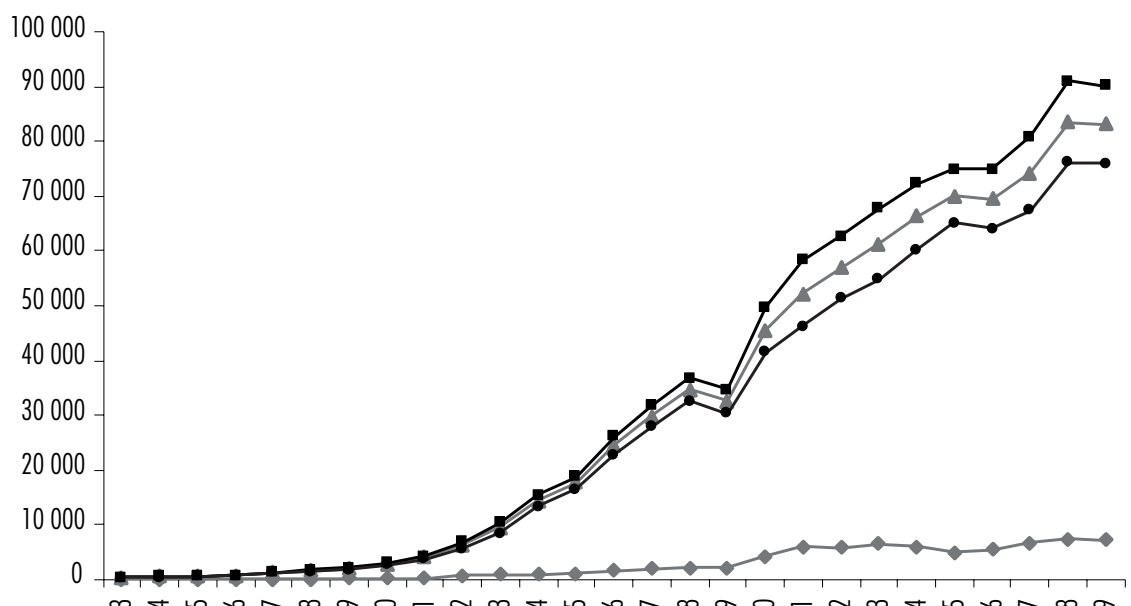

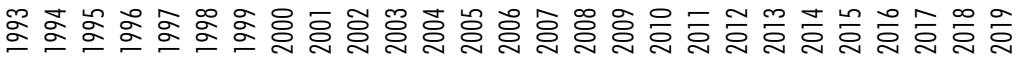

$\rightarrow$ Exportaciones $\rightarrow$ Importaciones $\rightarrow$ Suma $\rightarrow$ Déficit

Fuente: elaboración propia de acuerdo con las cifras de la SE (2020).

periodo de entre tres y cuatro años. Sin embargo, a partir de esa fecha, el lapso de la duplicación se prolongó aproximadamente a ocho años.

La figura 1 también muestra otra característica sobre los intercambios comerciales sostenidos entre China y México durante los últimos 26 años. Antes de 2000 , a pesar de ubicar en un proceso de crecimiento acelerado los volúmenes de cada uno de los indicadores, se encontraban en niveles reducidos, y además la brecha entre importaciones y exportaciones era reducida, menor a US\$2.0 mil millones por año. No obstante, para el periodo en el que la tasa de crecimiento de los intercambios comerciales se redujo a un dígito a partir de 2010, el saldo deficitario de México en su balanza comercial con China rebasó los US\$50 mil millones por año desde 2012. De 2010 a 2019, el saldo deficitario se amplió con un monto total de US\$34.5 miles de millones en términos absolutos, y el aumento neto en promedio de US\$3.8 mil millones por un año.

A partir de 2010, el aumento considerable de los flujos comerciales entre las dos economías, de las importaciones y en consecuencia del saldo deficitario, se explica por el impulso adicional derivado del ingreso de China a la OMC y por los nuevos arreglos realizados por ambas autoridades en materia del comercio bilateral, como se menciona líneas arriba. Por supuesto, existen 
otros elementos de los cuales no debe subestimarse su impacto y que están directamente relacionados con la reconfiguración de las cadenas globales del valor a raíz de las crisis financieras ocurridas en 2007 por un lado, y, por otro, el reforzamiento de las medidas de promoción sectorial instrumentadas por la SE con el propósito de recuperar y fortalecer la dinámica de las actividades exportadoras.

Las cifras estadísticas demuestran que México fue uno de los pocos países que recuperaron su nivel de exportación registrada antes de la crisis financiera hacia Estados Unidos de manera inmediata en 2010, después de sufrir caídas importantes en 2009, dinamismo que se mantuvo hasta 2019. De hecho, las ventas de productos mexicanos al mercado estadounidense son de tendencia creciente, al pasar de 238.7 en 2010 a 371.0 en 2019, ambos en términos de miles de millones de dólares (mmd), con un aumento neto de US\$132.3 mmd. Es decir, la coincidencia observada entre el ajuste de la estructura de los productos importados desde China con mayor proporción en el consumo generalizado hasta los de insumo, intermediarios y de capital, y el dinamismo registrado por las exportaciones mexicanas para Estados Unidos en el mismo lapso, sería difícil de catalogar como casual.

Es decir, el menor ritmo de crecimiento del comercio bilateral después de 2010 es válido cuando se mide en términos relativos; no obstante, en términos absolutos el panorama es diferente, pues la brecha entre exportaciones e importaciones se amplia cada vez más en favor del país asiático debido a la fuerte participación de China con su política de apertura y a la estructura de los eslabonamientos de las cadenas globales de valor. De manera que, para tener una descripción completa, se deben considerar las cifras tanto relativas como absolutas.

\section{Proporción entre importaciones y exportaciones con tendencia decreciente a pesar de las fluctuaciones persistentes}

Con el propósito de analizar la tendencia dinámica de los flujos comerciales efectuados por las dos economías y, sobre todo, de diagnosticar si existe estabilidad y convergencia dinámica o no, es importante mencionar que se observa con claridad un desequilibrio acentuado durante la última década en términos absolutos. Por ello, se plantea la opción de utilizar un término relativo que se formaliza mediante la proporción entre importaciones y exportaciones realizadas por México en su comercio con China. 
De este modo, si se expresan las importaciones mexicanas procedentes de China con M y las exportaciones de México hacia la economía asiática con X, la proporción $P x$ se define como:

$$
P x=M / X
$$

De acuerdo con las cifras estadísticas reveladas por la SE, que se presentan en la figura 1, el comportamiento de la serie derivada de la ecuación (1) se encuentra en la figura 2 .

Se observa que la serie $P x$ arroja una tendencia decreciente con fluctuaciones persistentes y cada vez de menor magnitud con el transcurso del tiempo en el lapso analizado; asimismo, en términos del promedio móvil de cinco ańos, el mismo indicador suaviza las fluctuaciones.

En la figura 2 también se puede apreciar que la variable $P x$ en algunos años llegó a ubicarse en 27.2, 19.8 y 17.0 en 1997, 1996 y en 2008, respectivamente, como niveles más altos; mientras que el valor más bajo se registró en 1993 y 2011 , de 8.6 y 8.8 en cada caso, con un promedio de 13.4 en todo el periodo. Es decir, por cada dólar de exportaciones mexicanas hacia China, las importaciones respectivas tenderían hacia US\$13.4. No obstante, el mismo indicador fue de aproximadamente 11 durante los últimos 10 ańos, cifra inferior al promedio arrojado durante todo periodo de análisis.

Figura 2. Proporción entre importaciones y exportaciones de México con China, 1993-2019

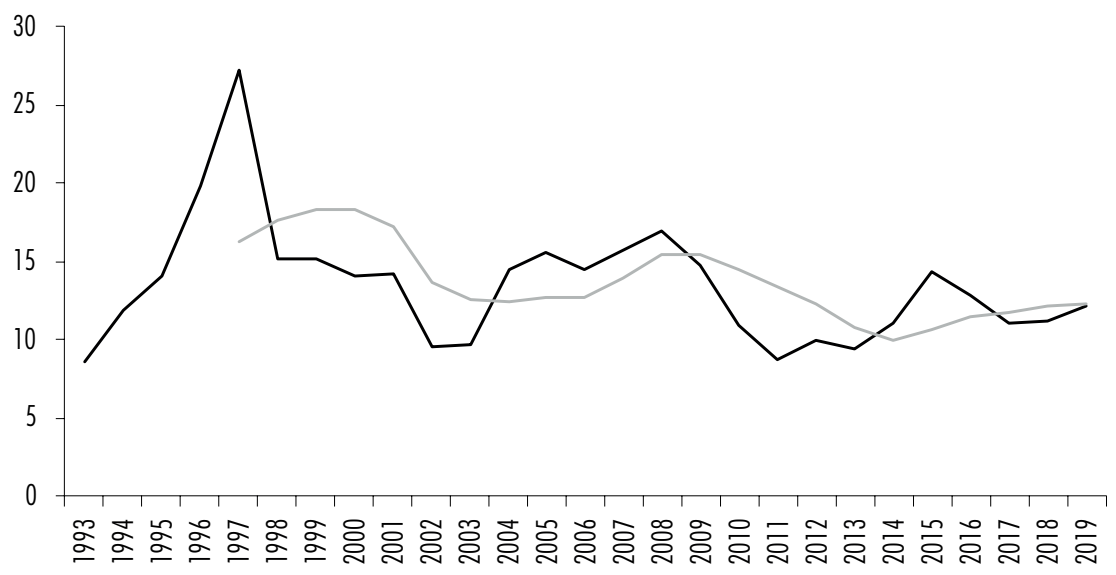

- M/X Promedio móvil

Fuente: elaboración propia de acuerdo con las cifras de la SE (2020). 
El comportamiento anterior del mismo indicador propuesto también se puede comprobar a través de calcular los promedios móviles $(M A)$ con el propósito de eliminar los efectos temporales causados por sucesos diversos. En ese caso, se aplica el $M A$ por cada cinco años (véase figura 2) cuyos resultados también registran una tendencia decreciente.

Asimismo, se calculan las varianzas de la serie $P x$ en sus distintas modalidades dentro de un lapso de 4, 8 y 10 ańos, respectivamente, cuyos resultados se encuentran en la figura 3.

Figura 3. Promedio móvil y varianzas de distintas modalidades de $P x$

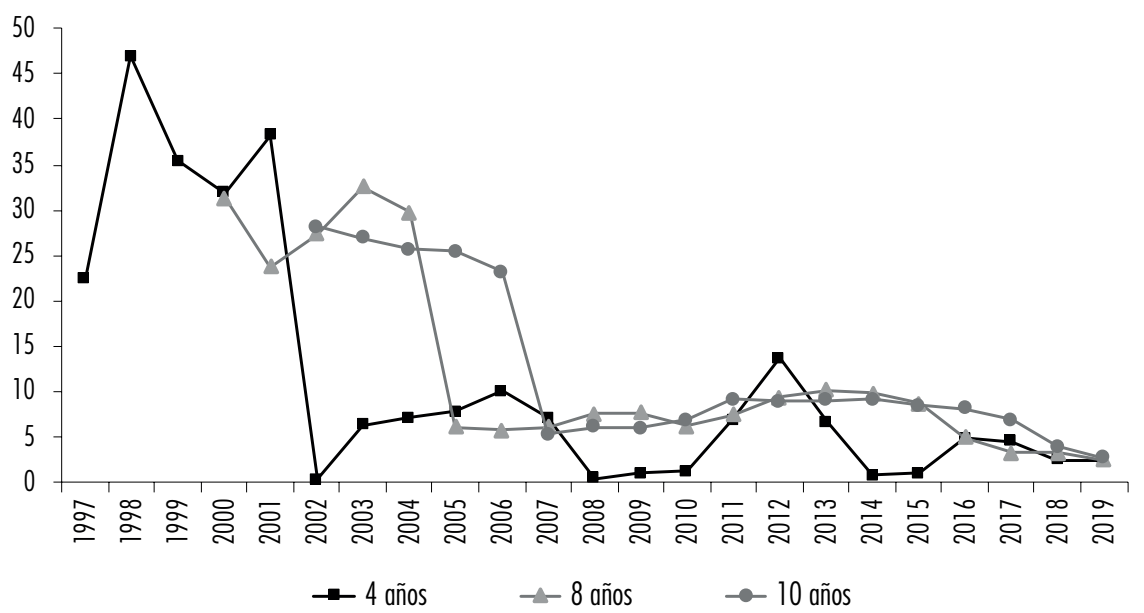

Fuente: elaboración propia de acuerdo con las cifras de la SE (2020).

Las varianzas en sus distintas modalidades apuntan a la misma dirección: una tendencia decreciente que, para el último año, las cifras son $2.41,2.42$ y 2.61 , respectivamente para rezagos de 4, 8 y 10 años. De esta manera, se constata que la serie $P x$ no sólo registra una tendencia decreciente, sino también fluctuaciones persistentemente reducidas.

\section{ANÁLISIS DE CONVERGENCIA AL EQUILIBRIO Y LA ESTABILIDAD DINÁMICA DE $P X$}

El análisis cualitativo anterior indica que el comercio bilateral entre China y México registra una tendencia convergente, sobre todo, cuando se utiliza la proporción entre importaciones y exportaciones realizadas por México con su 
segundo socio comercial, esto es, $P x$ en la ecuación (1). Para confirmar la posible convergencia al equilibrio y la estabilidad dinámica de la serie, se requiere aplicar una prueba de raíz unitaria.

\section{Convergencia al equilibrio o estabilidad dinámica de una serie}

Con fines de simplicidad, el análisis de estabilidad dinámica se empieza con un modelo de caminata aleatoria sin deriva visto como una ecuación en diferencias de primer orden:

$$
Y_{t}=a_{0} Y_{t-1}
$$

Lo cual se puede resolver a través del proceso de retrasos iterativos.

$$
Y_{t}=a_{0}^{t} Y_{0}
$$

Generalizando:

$$
Y_{t}=A b^{t}
$$

En la solución de la ecuación (2) del modelo de caminata aleatoria, la parte $A$ funge como una constante que solamente genera efecto de escala sin modificar la configuración básica del comportamiento de $b^{t}$. Por ello, la trayectoria discreta del tiempo que se presenta en la ecuación (4) depende principalmente del valor de $b$. Si la función $Y_{t}$ tendiera a cero cuando $t$ incrementa infinitamente, el equilibrio es dinámicamente estable e $Y_{t}$ converge al equilibrio; en caso contrario, la serie $Y_{t}$ sería divergente si $t$ aumentara de manera infinita.

En la tabla 1 se resume el comportamiento de la trayectoria dinámica de $Y_{t}$ de acuerdo con los valores de $b$.

Tabla 1. Trayectoria dinámica de $Y_{t}$

\begin{tabular}{cl}
\hline$b>0$ & No oscilatorio \\
$b<0$ & Oscilatorio \\
$|b| \geq 1$ & Divergente \\
$|b|<1$ & Convergente \\
\hline
\end{tabular}

Fuente: elaboración propia. 
Para un modelo representado por una ecuación en diferencias no homogénea y de primer orden que podría ser un modelo de caminata aleatoria con deriva, la expresión corresponde a la ecuación (5).

$$
Y_{t}+a Y_{t-1}=c
$$

Dónde $a$ y $c$ son constantes.

La solución general se compone por la suma de una solución particular $Y_{p}$ y una solución complementaria $Y_{c}$, las cuales forman una solución general para la ecuación no homogénea completa como se muestra en la ecuación (5).

De este modo, la solución complementaria estará en términos de la ecuación (2):

$$
Y_{t}+a Y_{t-1}=0
$$

Su función característica es:

$$
\begin{aligned}
& (b+a)=0 \\
& b=-a
\end{aligned}
$$

La solución complementaria es:

$$
Y=A(-a)^{t}
$$

Para hallar la solución particular, existen dos caminos que dependen del valor que toma $a$. Cuando $a \neq-1$, el primer tipo de solución particular de la ecuación (6) es:

$$
k=\frac{c}{1+a}
$$

Como indican Chiang y Wainwright (2006), si $a=-1$, la solución particular queda indefinida, y en términos del análisis del apartado anterior, representa un caso de raíz unitaria, por lo que es imperativo buscar otra solución particular para la ecuación no homogénea (ecuación 5). En este caso

$$
Y_{p}=c t
$$


De lo anterior, si $a \neq-1$, la solución general es:

$$
Y_{t}=A(-a)^{t}+\frac{c}{1+a}
$$

Para el caso en el que $a=-1$ la solución general es:

$$
Y=A(-a)^{t}+c t=A+c t
$$

Utilizando la condición inicial: si $t=0$ entonces $Y_{t}=Y$, para el caso de que $a \neq-1$,

$$
A=Y_{0}-\frac{c}{1+a}
$$

Por lo tanto, la ecuación (10) se sustituye por:

$$
Y_{t}=\left[Y_{0}-\frac{c}{1+a}\right](-a)^{t}+\frac{c}{1+a}
$$

Si $a=-1$, entonces la solución general es;

$$
Y_{t}=Y_{0}+c t
$$

Dónde $Y_{0}$ es el valor inicial de la serie.

Ante las dos soluciones generales representadas por las ecuaciones (12) y (13), se puede observar que para el caso de que $a=-1$, la trayectoria de $Y_{t}$ de acuerdo con la ecuación (13), no convergiría hacia ningún equilibrio por ser divergente al aumentar el valor de $t$ de manera infinita. Por su parte, cuando $a=1, Y_{t}$ adopta el valor de $A+y_{p}$ de acuerdo con la ecuación (12) que tampoco podrá alcanzar a $Y_{p}$ a menos que $A=0$. En consecuencia, la trayectoria se considera divergente, debido a que $A \neq 0 \mathrm{y}$, por lo tanto, habrá una desviación constante respecto al equilibrio móvil que es la solución particular, $\frac{c}{1+a}$ pero en ninguna circunstancia convergiría hacia ese punto de equilibrio.

Por ello, al estipular la condición de convergencia de la trayectoria de tiempo $Y_{t}$ hacia el equilibrio $Y_{p}$ debe descartar el caso de $b= \pm 1$, que en otras palabras se traduce en la presencia de una raíz unitaria. En consecuencia, se confirma la condición de equilibrio: que una trayectoria es convergente si y sólo si $|b|<1$. 
En resumen, la solución general de la ecuación (5) convergiría hacia su particular. $Y_{p}$ representa el nivel de equilibrio intertemporal de $Y_{t}$, e $Y_{c}$ son las desviaciones de la trayectoria dinámica respecto a ese equilibrio.

\section{Prueba de raíz unitaria}

Con el propósito de comprobar la existencia o no de la raíz unitaria de una serie temporal, con frecuencia se requiere la aplicación de la prueba de DickeyFuller Aumentada (1979), que es de gran importancia para determinar la estacionariedad de la serie o la ausencia de raíz unitaria (Granger y Newbold, 1974).

Sea que la ecuación siguiente representa un proceso estocástico sin deriva,

$$
Y_{t}=\rho Y_{t-1}+u_{t}
$$

Dónde $u_{t} \sim \operatorname{IIDN}\left(0, \sigma^{2}\right)$. Si $\rho=1$, se trata de un modelo de caminata aleatoria sin deriva y se tiene la presencia de raíz unitaria; o lo que es lo mismo, una serie no estacionaria. Si $|\rho|<1, Y_{t}$ se considera como una serie estacionaria y convergente.

No obstante, por medio de la prueba $t$ acostumbrada para comprobar la hipótesis de que $\rho=1$, podría derivar un sesgo muy marcado que desviaría el resultado (Gujarati y Porter, 2010). De esta manera, se procede a realizar ajustes sobre la ecuación (14) restando $Y_{t-1}$ de manera simultánea en ambos lados para obtener la ecuación (15).

$$
\begin{aligned}
& Y_{t}-Y_{t-1}=\rho Y_{t-1}-Y_{t-1}+u_{t} \\
& \Delta Y_{t}=(\rho-1) Y_{t-1}+u_{t} \\
& \Delta Y_{t}=\delta Y_{t-1}+u_{t}
\end{aligned}
$$

Dónde $\delta=(\rho-1)$ y $\Delta$ es el operador de primeras diferencias.

Por lo tanto, se aplica la estimación a la ecuación (15) y no a la ecuación (14) para comprobar la hipótesis nula de que $\delta=0$ contra la alternativa de $\delta<0$. 
- $H_{0}: \delta=0$ (que implica un proceso estocástico no estacionario o presencia de una raíz unitaria).

- $H_{1}: \delta<0$ (que implica un proceso estocástico estacionario) posiblemente alrededor de una tendencia determinista.

En la práctica, las anteriores pruebas se realizan en sus distintas modalidades al estimar la ecuación de regresión.

- $Y_{t}$ es una caminata aleatoria (sin intercepto): $\Delta Y_{t}=\delta Y_{t-1}+u_{t}$

- $Y_{t}$ es una caminata aleatoria con deriva (con intercepto): $\Delta Y_{t}=\beta_{1}+\delta Y_{t-1}+u_{t}$

- $Y_{t}$ es una caminata aleatoria con deriva alrededor de una tendencia determinista $\Delta Y_{t}=\beta_{1}+\beta_{2} t+\delta Y_{t-1}+u_{t}$, donde $t$ es la variable de tiempo o de tendencia.

En consecuencia, si no se rechaza la hipótesis nula $\delta=0$, entonces $\rho=1$; i.e., se comprueba la presencia de la raíz unitaria, y la serie $Y_{t}$ es no estacionaria.

Una vez que se obtienen los resultados de la prueba, antes de analizarlos se requiere decidir cuál de los tres modelos es el adecuado. Lo más evidente es descartar aquellos en los que el valor de $\delta$ es positivo. Pues en vista de que $\delta=(\rho-1)$, una $\delta$ positiva implicaría que $\rho>1$ y aunque es una posibilidad teórica (divergencia), su exclusión está basada en que la serie de tiempo $Y_{t}$ sería explosiva. $^{3}$

Es importante destacar dos puntos sobre el resultado de la prueba de hipótesis de raíz unitaria. En primer lugar, para los casos de una caminata aleatoria (sin intercepto) y de una caminata aleatoria con deriva (con intercepto), la estacionariedad de la serie $Y_{t}$ coinciden con la convergencia al equilibrio o la existencia de estabilidad dinámica de la misma. Sin embargo, para el tercer caso que implica una tendencia determinista, la serie $Y_{t}$ no necesariamente converge hacia un nivel del equilibrio. ${ }^{4}$

En esta prueba, un punto importante es descartar la posibilidad de que $\delta>0$ porque en este caso $\rho>1$, y de ser así, la serie de tiempo subyacente sería explosiva o divergente. Por ello, la prueba de raíz unitaria se limita en $\leq 1$.

4 Es importante mencionar que para un modelo representado por la ecuación (15), la no estacionariedad de $Y_{t}$, derivados de la prueba de raíz unitaria, no necesariamente implicaría la divergencia de la serie $Y_{t}$. Sin embargo, la comprobación tanto matemática como práctica no se analiza en este trabajo, pues se trata de una discusión de las consistencias entre estacionariedad y convergencia dinámica. 


\section{Aplicación de la prueba de raíz unitaria para el comercio bilateral entre China y México}

En esta sección, el análisis se aplica para cifras tanto anuales como mensuales de los intercambios comerciales sostenidos entre China y México, ambos con el propósito de comprobar la estacionariedad y posible convergencia dinámica a través de la utilización de las pruebas de raíz unitaria.

\section{Estimación para cifras anualizadas}

Para las cifras anualizadas, por medio del correlograma, se observa que los rezagos son estadísticamente significativos, pues tiene un valor de probabilidad menor que 0.05; en consecuencia, la serie es no estacionaria (véase figura 4).

Figura 4. Correlograma de la serie $P x$

\begin{tabular}{|c|c|c|c|c|c|c|c|c|}
\hline \multicolumn{2}{|c|}{ Autocorrelation } & \multicolumn{3}{|c|}{ Partial correlation } & $A C$ & PAC & Q-Stat & Prob \\
\hline 1 & $\square$ & 1 & $\square$ & 1 & 0.525 & 0.525 & 8.0296 & 0.005 \\
\hline 1 & 1 & $1 \square$ & 1 & 2 & 0.114 & -0.223 & 8.4273 & 0.015 \\
\hline 1 & 1 & 10 & 1 & 3 & -0.107 & -0.092 & 8.7874 & 0.032 \\
\hline $1 \square$ & 1 & । 드 & 1 & 4 & -0.208 & -0.110 & 10.222 & 0.037 \\
\hline $1 \square$ & 1 & । 드 & 1 & 5 & -0.253 & -0.125 & 12.438 & 0.029 \\
\hline $1 \square$ & 1 & 1 드 & 1 & 6 & -0.275 & -0.147 & 15.185 & 0.019 \\
\hline 1 & 1 & 1 & 1 & 7 & -0.008 & 0.257 & 15.187 & 0.034 \\
\hline
\end{tabular}

Fuente: estimación propia por medio del software econométrico E-views.

Aplicando pruebas de raíz unitaria para $P x$ anualizada, se tienen los siguientes resultados en cada una de sus tres distintas modalidades de regresión, a través del enfoque Dickey-Fuller Aumentada. Para los tres casos no se rechaza la hipótesis nula, dado que se obtuvieron valores de probabilidad de $0.4694,0.0798$ y 0.0803 , respectivamente, lo que indica que la serie es no estacionaria o tiene tenencia estocástica (las estimaciones se muestran en las tablas A1, A2 y A3 del Apéndice).

A pesar de los resultados obtenidos en las diferentes modalidades de regresión, las primeras dos regresiones presentan resultados convergentes para la serie $P x$, mientras que la última con tendencia explosiva (divergente) sin estabilidad dinámica es el modelo elegido para determinar su estabilidad de acuerdo con los criterios de Akaike, Schwartz y Hannan-Quinn. 


\section{Estimación para cifras mensuales}

Para las cifras mensuales, los resultados de la prueba de raíz unitaria afirman que la serie $P x$ es estacionaria y convergente.

Se considera la fluctuación mensual de los intercambios comerciales entre China y México, en el periodo 1993-2019, estimada por el cociente entre las importaciones realizadas por México desde China y las exportaciones mexicanas a China.

$\mathrm{Al}$ analizar la figura 5, en términos de estabilidad y estacionariedad, los resultados son más claros que en el caso de las cifras anuales (véase figura 2), de modo que, al tener valores de probabilidad de 0.000 en las tres modalidades de la regresión se rechaza la hipótesis nula de que la serie tiene una raíz unitaria (como se muestra en las tablas A4, A5 y A6 del Apéndice).

Asimismo, al ser menor el valor crítico del estadístico en comparación con lo observado, se rechaza la hipótesis nula, en sus distintos niveles de confianza, de 99, 95 y 90\%, respectivamente. De tal manera, la serie $P x$ con cifras mensuales desde 1993 hasta 2019 es un proceso estocástico estacionario. Por su parte, los coeficientes derivados de las regresiones representan valores negativos, los cuales confirman la no existencia de raíz unitaria de la misma variable, y de esta manera, también se trata de una serie con estabilidad dinámica.

\section{Figura 5. Proporción entre importaciones y exportaciones de México con China}

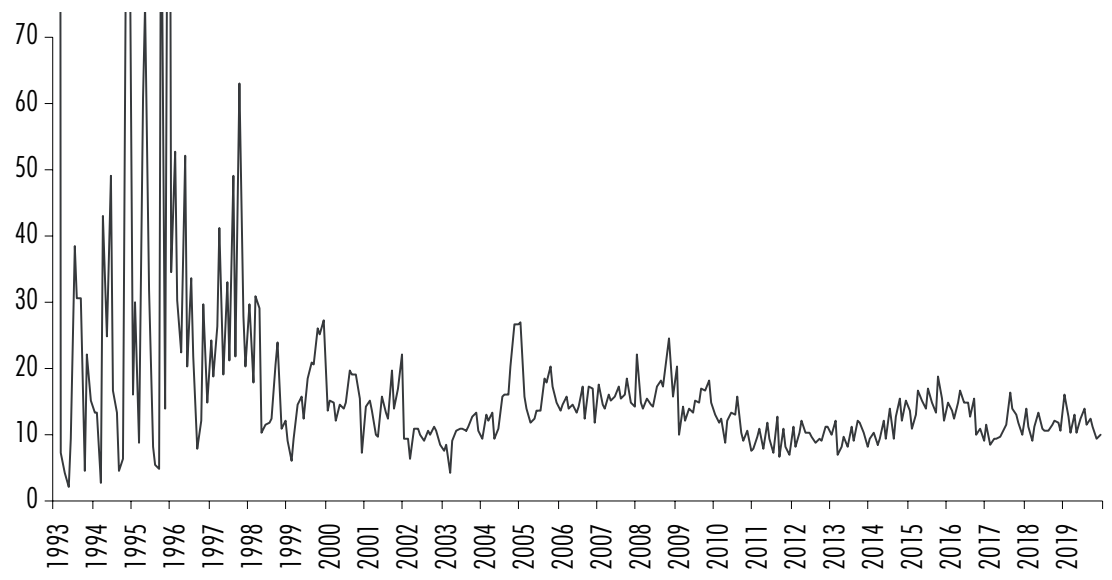

Fuente: elaboración propia de acuerdo con las cifras de la SE (2020). 


\section{Pronósticos basados en las pruebas de raíz unitaria}

De acuerdo con los resultados enlistados en la tabla A5 del Apéndice sobre la prueba de raíz unitaria, se tiene la siguiente ecuación en diferencias:

$$
Y_{t}-0.388238 \delta Y_{t-1}=10.69714
$$

La solución general de la ecuación (16) consiste en la suma de la parte complementaria y la particular.

$$
Y_{t}=A(0.388238)^{t}+17.485787
$$

En este sentido, la solución de la ecuación se traduce en que la proporción entre importaciones y exportaciones realizadas por México con su segundo socio comercial converge en 17.485787 cuando se proyecta hacia el futuro; es decir, por cada dólar de exportaciones mexicanas hacia China las importaciones recíprocas tenderían hacia su nivel del equilibrio, de \$USD17.5 (véase figura 6).

Es pertinente señalar que la convergencia en el punto señalado de US\$17.5 se refiere específicamente a que, a pesar de las fluctuaciones de las importaciones y exportaciones entre México y China, la proporción es la misma; dicho en otras palabras, estas dos variables tienen una trayectoria de comportamiento correspondiente al moverse en el mismo sentido y proporción, de manera que el cociente entre ellas tenderá a 17.5. No obstante, no se debe confundir con el saldo de la balanza comercial en términos absolutos que representa la diferencia entre las dos variables con una tendencia explosiva.

Cabe mencionar que durante los primeros dos meses de 2020, el indicador analizado en este estudio empezó a registrar valores de 14.3 y 12.6, respectivamente, a pesar de las reducciones e incluso estabilidades observadas durante los últimos 10 ańos. Por ello, es muy probable que 2020 represente el inicio de un cambio estructural basado en hechos registrados, tales como las fricciones comerciales entre China y Estados Unidos, y la pandemia causada por SARsCov-2, los cuales se requiere analizar con mayor detalle en el futuro. 
Figura 6. Convergencia de la proporción entre importaciones y exportaciones de México con China

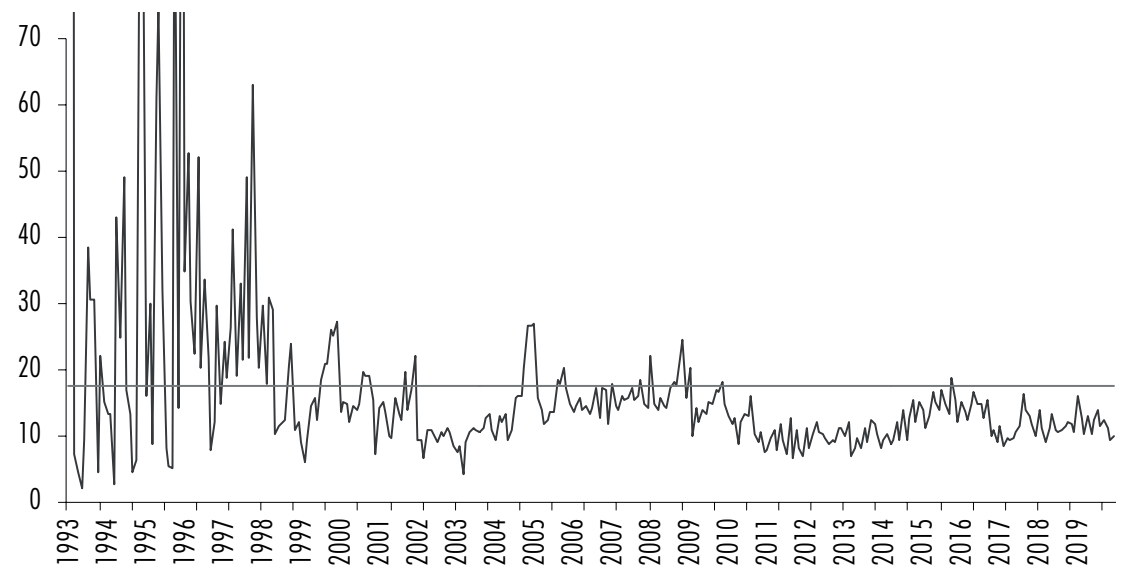

Fuente: elaboración propia de acuerdo con las cifras de la SE (2020).

\section{CONCLUSIONES}

Desde el punto de vista cuantitativo, se puede concluir que a pesar de las fluctuaciones persistentes registradas por los intercambios comerciales entre China y México, la varianza de ellos se ha visto reducida a lo largo de los últimos 26 años. Además, las pruebas de raíz unitaria confirman que los intercambios comerciales entre ambos países en términos relativos tienden a la convergencia en un nivel de estabilidad de aproximadamente 17.5, desde su nivel máximo de 27.2 observado en 1997, independientemente del saldo absoluto creciente.

De acuerdo con las cifras anuales, la estabilidad de la serie resultaría ambigua, ya que no se trata de una serie estacionaria al no poder rechazar la hipótesis nula de la prueba de raíz unitaria. Por ello se procedió la aplicación de cifras mensuales, que confirma la estacionariedad y la convergencia dinámica hacia el nivel del equilibrio.

Los resultados obtenidos se traducen en que, a pesar de las fluctuaciones, los intercambios comerciales sino-mexicanos convergen en 17.5; es decir que, por cada dólar que México exporta hacia el país asiático, se importan US\$17.5, lo que ratifica el proceder de China en torno a sus reformas de apertura e implicaciones que tiene sobre la posición de México en el comercio exterior.

Para efectos de esta investigación, y en función de los resultados obtenidos en el periodo de estudio, se puede afirmar que el incremento de la demanda 
por productos de origen chino pone de manifiesto el incremento de manera constante y alcista del saldo comercial en la relación México-China en favor de este último en todo el periodo, de manera que el gigante asiático se consolida como el segundo socio comercial de México.

Ante ello, resulta imperativo identificar las potencialidades en las relaciones sino-mexicanas y sus posibles impactos en su comercio con Estados Unidos, de manera que sea posible implantar un nuevo marco institucional capaz de replantear el comercio internacional en términos de política comercial e incentivos sectoriales que permitan incrementar la competitividad del país, reestructurar las cadenas globales de valor y estimular los sectores de producción manufacturera y tecnológicos clave y en los que China ha ganado terreno.

Bajo este contexto, el reto que enfrentan la economía mexicana y la fuerza exportadora nacional no sólo consiste en el fortalecimiento y mejoramiento del nivel de competitividad general, sino también en el desarrollo eficiente de los eslabonamientos locales e integración sectorial de la industria manufacturera. De tal manera, se pueden incorporar cada vez más los contenidos nacionales y aumentar en consecuencia, la participación del valor agregado dentro de las exportaciones, aprovechando los nuevos marcos del desarrollo derivados del nuevo acuerdo comercial en la región norteamericana.

\section{APÉNDICE}

Tabla Al. Prueba de Dickey-Fuller Aumentada para el modelo de caminata aleatoria con cifras anualizadas

\begin{tabular}{|c|c|c|c|}
\hline \multicolumn{4}{|c|}{ Prueba de Dickey-Fuller Aumentada. Variable Dependiente D(PX) } \\
\hline & Coeficiente & Estadístico $t$ & Valor_p \\
\hline $\operatorname{Px}(-1)$ & -0.0290 & -0.5480 & 0.5887 \\
\hline \multicolumn{4}{|c|}{ Hipótesis nula: Px tiene una raíz unitaria } \\
\hline & & Estadístico $t$ & Valor_p \\
\hline & & -0.5480 & 0.4694 \\
\hline Prueba DFA & $1 \%$ & -2.6607 & \\
\hline \multirow[t]{2}{*}{ Valores críticos } & $5 \%$ & -1.9550 & \\
\hline & $10 \%$ & -1.6090 & \\
\hline
\end{tabular}

Fuente: elaboración propia (resultados arrojados por el software econométrico E-views). 
Tabla A2. Prueba de Dickey-Fuller Aumentada para el modelo de caminata aleatoria con intercepto con cifras anualizadas

Prueba de Dickey-Fuller Aumentada. Variable Dependiente D(Px)

\begin{tabular}{|c|c|c|c|}
\hline & Coeficiente & Estadístico $t$ & Valor_p \\
\hline $\operatorname{Px}(-1)$ & -0.4684 & -2.7511 & 0.0114 \\
\hline C & 6.4809 & 2.6860 & 0.0132 \\
\hline \multicolumn{4}{|c|}{ Hipótesis nula: Px tiene una raíz unitaria } \\
\hline & & Estadístico ${ }^{\prime}$ & Valor_p \\
\hline & & -2.7511 & 0.0798 \\
\hline Prueba DFA & $1 \%$ & -3.7240 & \\
\hline \multirow[t]{2}{*}{ Valores críticos } & $5 \%$ & -2.9862 & \\
\hline & $10 \%$ & -2.6326 & \\
\hline
\end{tabular}

Fuente: elaboración propia (resultados arrojados por el software econométrico E-views).

Tabla A3. Prueba de Dickey-Fuller Aumentada para el modelo de caminata aleatoria con intercepto y tendencia con cifras anualizadas

Prueba de Dickey-Fuller Aumentada. Variable Dependiente D(PX)

\begin{tabular}{rccc}
\hline & Coeficiente & Estadistico $t$ & Valor_p \\
\hline Px(-1) & -0.5746 & -3.3572 & 0.0028 \\
$C$ & 10.2100 & 3.3714 & 0.0028 \\
Tendencia & -0.1747 & -1.8814 & 0.0732 \\
\hline
\end{tabular}

Hipótesis nula: Px tiene una raíz unitaria

\begin{tabular}{lccc}
\hline & Estadístico ${ }^{\circ}$ & Valor_p \\
\hline & & -3.3572 & 0.0803 \\
Prueba DFA & $1 \%$ & -4.3743 & \\
Valores críticos & $5 \%$ & -3.6032 & \\
& $10 \%$ & -3.2380 & \\
\hline
\end{tabular}

Fuente: elaboración propia (resultados arrojados por el software econométrico E-views). 
Tabla A4. Prueba de Dickey-Fuller Aumentada para el modelo de caminata aleatoria con cifras mensuales

Prueba de Dickey-Fuller Aumentada. Variable Dependiente D(PX)

\begin{tabular}{cccc}
\hline & Coeficiente & Estadistico $t$ & Valor_p \\
\hline $\operatorname{Px}(-1)$ & -0.4639 & -10.9959 & 0.0000 \\
\hline
\end{tabular}

Hipótesis nula: Px tiene una raíz unitaria

\begin{tabular}{lccc}
\hline & Estadistico t & Valor_p \\
\hline & & -10.9959 & 0.0000 \\
Prueba DFA & $1 \%$ & -2.5724 & \\
Valores críticos & $5 \%$ & -1.9418 & \\
& $10 \%$ & -1.6160 & \\
\hline
\end{tabular}

Fuente: elaboración propia (resultados arrojados por el software econométrico E-views).

Tabla A5. Prueba de Dickey-Fuller Aumentada para el modelo de caminata aleatoria con intercepto con cifras mensuales

\begin{tabular}{|c|c|c|c|c|}
\hline \multicolumn{5}{|c|}{ Prueba de Dickey-Fuller Aumentada. Variable Dependiente D(Px) } \\
\hline & & Coeficiente & Estadístico $t$ & Valor_p \\
\hline & $\operatorname{Px}(-1)$ & -0.6117 & -13.2227 & 0.0000 \\
\hline & C & 10.6971 & 6.2686 & 0.0000 \\
\hline \multicolumn{5}{|c|}{ Hipótesis nula: Px tiene una raíz unitaria } \\
\hline & & & Estadístico $t$ & Valor_p \\
\hline & & & -13.2227 & 0.0000 \\
\hline Prueba DFA & & $1 \%$ & -3.4512 & \\
\hline \multirow{2}{*}{\multicolumn{2}{|c|}{ Valores críticos }} & $5 \%$ & -2.8706 & \\
\hline & & $10 \%$ & -2.5716 & \\
\hline
\end{tabular}

Fuente: elaboración propia (resultados arrojados por el software econométrico E-views). 
Tabla A6. Prueba de Dickey-Fuller Aumentada para el modelo de caminata aleatoria con intercepto y tendencia con cifras mensuales

\begin{tabular}{|c|c|c|c|c|}
\hline \multicolumn{5}{|c|}{ Prueba de Dickey-Fuller Aumentada. Variable Dependiente $D(P x)$} \\
\hline & & Coeficiente & Estadístico $t$ & Valor_p \\
\hline & $\operatorname{Px}(-1)$ & -0.6520 & -13.6949 & 0.0000 \\
\hline & C & 19.3301 & 5.7965 & 0.0000 \\
\hline & Tendencia & -0.0504 & -2.9997 & 0.0029 \\
\hline \multicolumn{5}{|c|}{ Hipótesis nula: Px tiene una raíz unitaria } \\
\hline & & & Estadistico $t$ & Valor_p \\
\hline & & & -13.6949 & 0.0000 \\
\hline Prueba DFA & & $1 \%$ & -3.9878 & \\
\hline \multirow{2}{*}{\multicolumn{2}{|c|}{ Valores críticos }} & $5 \%$ & -3.4243 & \\
\hline & & $10 \%$ & -3.1352 & \\
\hline
\end{tabular}

Fuente: elaboración propia (resultados arrojados por el software econométrico E-views).

\section{BIBLIOGRAFÍA}

Anguiano, E. (2016). La relación México-China: desempeño y propuestas para el periodo 2016-2018. En E. Dussel (coord.). La relación MéxicoChina. Desempeño y propuestas para 2016-2018 (pp. 14-17). UnAm.

Cárdenas, L. y Dussel, E. (2011). El comercio intra-industrial en México: un comparativo entre China y Estados Unidos. Revista Comercio Exterior, 61(4). https://www.dusselpeters.com/49.pdf

Chiang, A. y Wainwright, K. (2006). Métodos fundamentales de economía matemática. McGraw-Hill.

De la Cruz, J. L. y Veintimilla, V. (2014). Evaluación y resultados económicos del tLCan, en el marco de la competencia comercial con China. En A. Oropeza (coord.). TLCAN 20 años. ¿Celebración, desencanto o replanteamiento? (pp. 479-504). UNAM, IIJU.

Diario Oficial de la Federación (DOF) (2007, 15 de agosto). Acuerdo por el que se da a conocer el protocolo de adhesión de la República Popular China a la Organización Mundial del Comercio. https://www.gob.mx/cms/ uploads/attachment/file/50776/A430.pdf 
Dickey, D. A. y Fuller, W. A. (1979). Distribution of estimators for autoregressive time series with a unit root. Journal of the American Statistical Association, 74(366). https://doi.org/10.2307/2286348

Dussel, E. y Gallagher, K. (2014). El huésped no invitado del TLCAN: China y la desintegración del comercio en América del Norte. En A. Oropeza (coord.). TLCAN 20 años. ¿Celebración, desencanto o replanteamiento? (pp. 441-478). UNAM, IIJU.

Embajada de México en China (2015). Relación Económica. https://embamex.sre.gob.mx/china/index.php/es/la-embajada/relacion-economica

Enders, W. (2015). Applied Econometrics Time Series. Wiley.

Espinosa, E. y Serra, J. (2005). Diez años del Tratado de Libre Comercio de América del Norte. En P. García Alba et al. (coords.). El nuevo milenio mexicano. Tomo I. México en el mundo. Editorial EON.

Gallagher, K. y Porzecanski, R. (2010). The dragon in the room. China and the future of Latin America industrialization. Stanford University Press.

y Dussel, E. (2013). China's economic effects on the U.S.-Mexico trade relationship: Towards a new triangular relationship? En E. Dussel, A. Hearn y H. Shalken (eds.). China and the new triangular relationships in the Americas (pp. 13-23). Centro de Estudios China-México.

Granger, C. W. y Newbold, P. (1974). Spurious regressions in econometrics. Journal of Econometrics, 2(2). https://doi.org/10.1016/0304-4076 (74) 90034-7

Guerrero, C. (2011). Introducción a la econometría aplicada. Trillas.

Gujarati, D. y Porter, D. (2010). Econometría. McGrawHill.

Harvey, A. C. (1993). Time series model. Harvester Wheatsheaf.

Jenkins, R. y Dussel, E. (2009). China and Latin America. Economic relations in the twenty-first century. DIE/UNAM-CECHIMEX.

Ley, S. (2012). Algunas reflexiones sobre el futuro de la relación MéxicoChina. En E. Dussel (coord.). 40 años de la relación entre México y China, acuerdos, desencuentros y futuro (pp. 59-64). UNAM.

Limas, A. (2019). Evolución y perspectiva de la relación económica MéxicoChina. En A. Vargas, A. Girón et. al. (coords.). China y México: 45 años de relaciones diplomáticas y culturales (pp. 81-87). UNAM.

Liu, X. (2012). Tipo de cambio peso-yuan y transacción comercial entre China y México, 1981-2001. Matices del Posgrado Aragón, (18). https:// publicaciones-aragon.unam.mx/repositorio/matices/18/18.pdf

López, J. A. y Rodil, O. (2019). La inserción económica de China en Latinoamérica. Investigación Económica, 78(219). http://dx.doi.org/10.22201/ fe.01851667p.2019.310.71549 
Maddala, G. S. (2001). Introduction to Econometrics. Wiley.

Oropeza, A. (2006). China entre el reto y la oportunidad. UNAM, IIJ.

Ortiz, A. (2011). El superávit comercial de México con Estados Unidos. Problemas del Desarrollo. Revista Latinoamericana de Economía, 164(42). http://www.scielo.org.mx/pdf/prode/v42n164/v42n164a8.pdf

Ruíz, P. (2004). El tLCan y la balanza comercial de México. Economía Infor$m a$, (327). http://www.economia.unam.mx/publicaciones/reseconinfor$\mathrm{ma} / \mathrm{pdfs} / 327 / 05 \mathrm{Napoles} . \mathrm{pdf}$

Secretaría de Economía (SE) (2007). Acuerdo por el que se da a conocer el protocolo de adhesión de la República Popular China a la Organización Mundial del Comercio. https://www.gob.mx/cms/uploads/attachment/ file/50776/A430.pdf

(2008). Vigente acuerdo México-China en materia de cuotas compensatorias. http://www.2006-2012.economia.gob.mx/eventos-noticias/sala-deprensa/comunicados/5982-vigente-acuerdo-mxicochina-en-materia-decuotas-compensatorias

(2020). Comercio Exterior/Estadísticas Históricas. https://www. economia.gob.mx/files/gobmx/comercioexterior/estadisticas/Anual-Importa-2019.pdf y https://www.economia.gob.mx/files/gobmx/comercioexterior/estadisticas/Anual-Exporta-2019.pdf.

United States Census Bureau (2015). Seasonally Adjusted (by Geography) https://www.census.gov/foreign-trade/Press-Release/current_press_release/index.html

Villareal, R. (2014). México es un laberinto: ¡cómo replantear el modelo económico en una era post TLCAN? En A. Oropeza (coord.). TLCAN 20 años. ¿Celebración, desencanto o replanteamiento? (pp. 81-94). UNAM, IIJ.

Zhang, K. (2013). China y México: una nueva etapa en las relaciones bilaterales. Orientando. Temas de Asia Oriental. Sociedad, Cultura y Economia, (6). https://orientando.uv.mx/index.php/orientando/article/view/878/1615

Zottele, A. C. y Santiago, M. A. (2015). Relaciones comerciales de México con el sudeste asiático: acuerdos y actores sociales. Portes. Revista Mexicana de estudios sobre la Cuenca del Pacifico, 9(7). http://revistasacademicas.ucol. $\mathrm{mx} /$ index.php/portes/article/view/369/313 